\title{
Upward propagating high-frequency Alfvén waves as identified from dynamic wave-like spicules observed by SOT on Hinode
}

\author{
J.-S. He ${ }^{1,2}$, C.-Y. Tu${ }^{1}$, E. Marsch ${ }^{2}$, L.-J. Guo ${ }^{1}$, S. Yao ${ }^{1}$, and H. Tian ${ }^{1,2}$ \\ 1 Department of Geophysics, Peking University, Beijing, PR China \\ e-mail: [chuanyitu; jshept] @pku.edu.cn \\ 2 Max-Planck-Institut für Sonnensystemforschung, Katlenburg-Lindau, Germany \\ e-mail: marsch@mps.mpg.de
}

Received 10 August 2008 / Accepted 6 February 2009

\begin{abstract}
Aims. We identify high-frequency Alfvén waves propagating upward in the solar chromosphere and transition region from observation by Solar Optical Telescope (SOT) onboard Hinode.

Methods. The spicule shape is enhanced through application of a normal radial gradient filter and an un-sharp mask on the images taken by SOT. The displaced position of the spicule is at each height obtained by tracing the maximum intensity after image processing. The dominant wave period is obtained by the FFT method applied to the time variations of the displaced position at a certain height. The phase speed is estimated with the help of a cross-correlation analysis of two temporal sequences of the displaced positions at two heights along the spicule.

Results. We find in four cases that the spicules are modulated by high-frequency $(\geq 0.02 \mathrm{~Hz})$ transverse fluctuations. Such fluctuations are suggested to be Alfvén waves that propagate upward along the spicules with phase speed ranges from 50 to $150 \mathrm{~km} \mathrm{~s}^{-1}$. Three of the modulated spicules show clear wave-like shapes with short wavelengths less than $8 \mathrm{Mm}$.

Conclusions. Our work identified directly upward propagation of Alfvén waves in the solar chromosphere and transition region. In addition to the recently reported Alfvén waves with very long wavelength and wave period, we find here four examples of Alfvén waves with shorter wavelengths and periods. These findings shed new light on the wave origin and on coronal and solar-wind heating.
\end{abstract}

Key words. waves - Sun: chromosphere - Sun: transition region - Sun: solar wind

\section{Introduction}

Upward propagating Alfvén waves are generally assumed to exist in the lower solar atmosphere and exploited in theoretical models for heating of the corona and solar wind acceleration (see, e.g., the reviews by Tu \& Marsch 1995; Hollweg \& Isenberg 2002). The models may basically be divided into two kinds. One kind of model assumes high-frequency Alfvén waves with the cyclotron damping mechanism (e.g., Tu \& Marsch 1997; Marsch \& Tu 1997; Axford et al. 1999; Tu \& Marsch 2001; He et al. 2008). The other kind of model assumes lowfrequency Alfvén waves which are subject to a nonlinear damping mechanism (e.g., Matthaeus et al. 1999; Hollweg 2000; Suzuki \& Inutsuka 2005; Cranmer et al. 2007). However, until recently it was difficult to identify directly the properties of Alfvén waves in the solar atmosphere from optical observations.

An indirect piece of evidence of the existence of Alfvén waves or turbulence is the extraordinary, besides the thermal motion effect, Doppler broadening of emission lines when observed on the disk (see the review by Wilhelm et al. 2007) and off the solar limb (e.g., Tu et al. 1998). The existence of Alfvén waves in the coronal plasma has been recently reported by Tomczyk et al. (2007), a result which is based on crosscorrelation analysis of Fe XIII (1074.7 nm) Line-of-Sight (LOS) Doppler velocity time series. That Alfvén waves exist in the chromosphere has been recently revealed by De Pontieu et al. (2007a), with the evidence coming from the transverse oscillation of spicules as observed in Ca II H (3968 ^) by SOT
(Tsuneta et al. 2008) onboard Hinode (Kosugi et al. 2007). In the work of De Pontieu et al. (2007a), Alfvén waves are thought to permeate the entire chromosphere, as inferred from the ubiquity of spicule oscillations, and the velocity amplitude of the oscillation is estimated to be on the order of 10 to $25 \mathrm{~km} \mathrm{~s}^{-1}$, and periods range from 100 to $500 \mathrm{~s}$. It was suggested that the spectrum of the generated Alfvén waves includes signals of both the photospheric 5-min p-modes and chromospheric 3-min oscillations, and that these results strongly support coronal heating models that are based on the dissipation of low-frequency waves.

De Pontieu et al. (2007a) found that the distribution of measured transverse displacements of 94 observed spicules agrees well with the distribution derived from a Monte Carlo simulation in which spicules carry low-frequency Alfvén waves with periods randomly chosen from a uniform distribution between 150 and $350 \mathrm{~s}$ and velocity amplitudes from a Gaussian distribution around $20 \pm 5 \mathrm{~km} \mathrm{~s}^{-1}$. The wavelength for their low-frequency Alfvén waves is reported to be generally in excess of the lengths of the field-tracing spicules, and the wavelength lower limit on the order of $20 \mathrm{Mm}$ is compatible with the value resulting from multiplication of the phase speeds $\left(50-200 \mathrm{~km} \mathrm{~s}^{-1}\right)$ with the long wave periods (100-500 s). However, Alfvén waves with short periods less than $50 \mathrm{~s}$ and short wavelength less than $10 \mathrm{Mm}$ have not yet been detected and studied from spicule dynamics. De Pontieu et al. (2007a) also reported the transverse displacement propagation with a phase speed on the order of $100-200 \mathrm{~km} \mathrm{~s}^{-1}$. However, it was not mentioned in their paper 

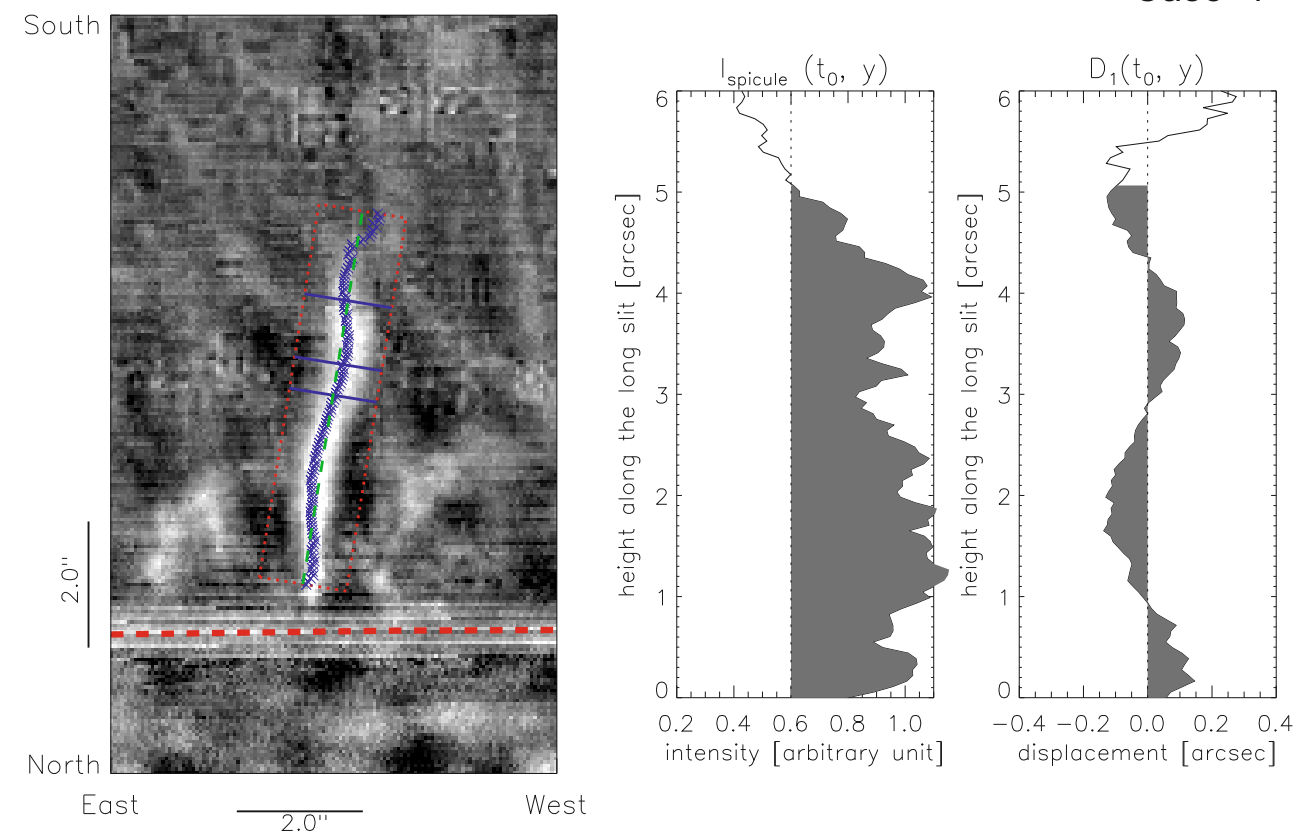

Fig. 1. Left panel: processed image obtained by SOT in Ca II H (3968 $\AA$ ) at $t_{0}=11: 40: 29$ UT. The red dotted box indicates the position of the spicule. The blue line is used to represent the skeleton of the spicule shape, $D_{0}\left(t_{0} ; y\right)$, which appears wave-like. Three blue short lines indicate the locations on the spicule where its displacement is further analyzed, i.e. Point-I, Point-II and Point-III. The dashed green line is the center longitudinal axis of the red dotted box. Middle panel: profile of the defined spicule intensity $I_{\text {spicule }}\left(t_{0} ; y\right)$. Right panel: profile of the spicule displacement $D_{1}\left(t_{0} ; y\right)$.

whether the propagating transverse displacement oscillates, like previously discussed 94 displacement oscillations just at a special height above the solar limb. De Pontieu et al. (2007a) modeled a mix of upward and downward propagating Alfvén waves on a spicule and compared the modeling result with the dynamics of one observed spicule. However, they did not show directly whether the Alfvén waves are propagating upward, downward or are a superposition of both.

In this paper, we present four cases showing upward propagating Alfvén waves identified from dynamic spicules observed by SOT on Hinode in Ca II H (3968 A). The wavelengths and periods of these waves are shorter than the ones previously reported. The wavelengths of Alfvén waves in our study are short enough to modulate the spicules such that they are looking wavelike. The periods of the wave in our study are short enough to be less than the time the spicules are visible. We speculate that the chromospheric waves identified here are generated by magnetic reconnection, and are at a frequency susceptible to cyclotron damping in the outer corona - a candidate mechanism for solar wind heating (e.g., Tu \& Marsch 1997).

\section{Observations and data analysis}

The dataset used here of image sequences in the Ca II H (3968 $⿱$ ) wavelength range was obtained by Hinode/SOT from 11:29:32 to 11:49:50 UT on 19 March 2007. During this period, SOT was looking at the southern polar region. The cadence for the image sequence is $4.8 \mathrm{~s}$. The spatial pixel size is $0.054^{\prime \prime}$. Such high temporal and spatial resolution allows us to observe the refined structure and dynamics of spicules in the polar region, which is a precondition to analyze an Alfvén wave carried by the spicule.

The SOT standard program "fg_prep.pro" is used to reduce the images by removing dark current and other camera artifacts. The jitter of the images introduced by the spacecraft is mostly removed by the correlation tracker system on SOT with trivial jitter of $0.01^{\prime \prime}(\sigma)$ remaining in the co-aligned time series (Shimizu et al. 2008). The time series have a slow pointing drift, with an average speed component of $\sim 0.017^{\prime \prime} / \mathrm{min}$ toward the north as identified from the solar limb slow drift in the original time series. This slow drift speed component is eliminated before studying the spicule dynamics. While the slow drift component tangential to the solar limb, which cannot be identified from the dynamically evolving time series, is left to be eliminated by subtracting the averaged linear transverse motion from the spicule dynamics in the following analysis. The dataset, after taking into account the above effects, is ready to measure the high-frequency displacement oscillation with amplitude being not less than $0.05^{\prime \prime}$. We take two steps of image processing in order to enhance the spicules for the convenience of further analysis. In the first step, the normal radial gradient filtering (NRGF) technique (Morgan et al. 2006) is implemented to filter the background radiation intensity, which fades away gradually along the radius. The purpose of NRGF is to enhance the structures in the off-limb region as compared with the structures on the solar disk. In the second step, the un-sharp mask technique (with "gradient" being the second-order gradient) (Koutchmy \& Koutchmy 1989) is applied to remove the diffuse strong radiation intensity in the off-limb region. Thus the spicule outline becomes clearer after application of the un-sharp mask.

We then select four spicules to analyze their shapes and dynamics. A long slit with a certain width, which is generally parallel to the spicule, is placed in the image sequences, see the left panel in Fig. 1 for the first case of spicule at the time $\left(t_{0}=\right.$ 11:40:29 UT). Such slit is used to record the intensity distribution (after the image processing) along and across the spicule, $I(x, y)$, where $x$ is the transverse coordinate in the slit, and $y$ is the longitudinal coordinate. The area of the slit is divided into $M \times N$ pixels, where $M$ is the number of columns (the number of pixels in the transverse coordinate) and $N$ is the number of rows 

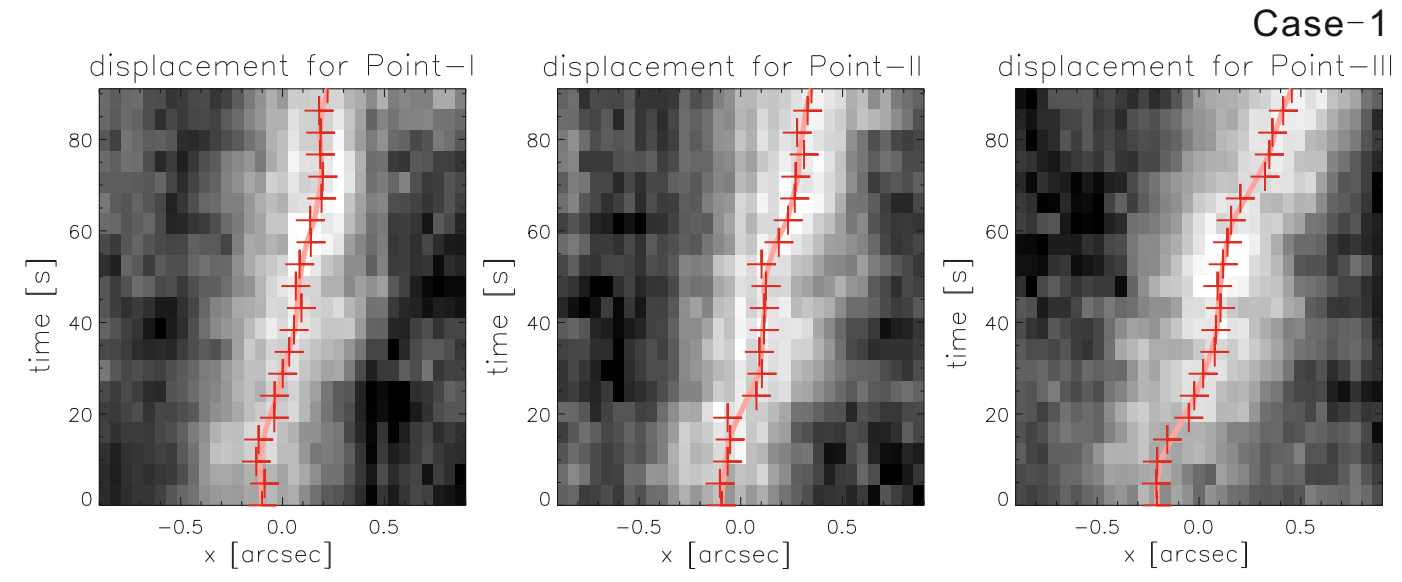

Fig. 2. From left through middle to right: profiles of $D_{0}(t, y)$ for Point-I, Point-II and Point-III, respectively, which are located at three heights marked with three blue short perpendicular slits listed from bottom to top in the left panel of Fig. 1.

(the number of pixels in the longitudinal coordinate). The transverse and longitudinal side lengths of the pixel, $\mathrm{d} x$ and $\mathrm{d} y$, are both set as $0.054^{\prime \prime}$. The spicule intensity of the displaced point in the $n$th row on the spicule at the time $t_{0}, I_{\text {spicule }}\left(t_{0}, y_{n \text {th row }}\right)$, is assigned with the mean of the intensity of the pixels having an intensity in the top 20 percent of the $M$ pixels in the $n$th row.

The intensity distribution along the spicule, $I_{\text {spicule }}\left(t_{0}, y\right)$, is shown in the middle panel of Fig. 1. The transverse position of the displaced point in the $n$th row on the spicule at the time $t_{0}$, $D_{0}\left(t_{0}, y_{n \text {th row }}\right)$, is set with mean position of the $M$ pixels with square intensity of every pixel as the weight. The transverse position obtained in this way has a sub-pixel resolution $\left(<0.054^{\prime \prime}\right)$. Thus, a transverse position profile as a function of the longitudinal coordinate of the slit, $D_{0}\left(t_{0}, y\right)$, can be obtained and is illustrated as a blue line in the left panel of Fig. 1, which appears wave-like relative to the central longitudinal axis of the long slit shown as a green dashed line in the figure. The spicule may be inclined off the longitudinal axis of the fixed slit during its evolution. The spatially oblique trend of the spicule at the time $t, D_{t}(y)$, is a linear-fitting result of $D_{0}(t ; y)$ at the time $t$. We subtract $D_{t}(y)$ from $D_{0}(t ; y)$, and obtain a modified displacement spatial profile at the time $t$, which is defined as $D_{1}(t ; y)$. We illustrate $D_{1}\left(t_{0}, y\right)$ in the right panel of Fig. 1. As can be seen in Fig. 1, the spicule appears wave-like in shape with a wavelength of $\sim 2100 \mathrm{~km}\left(3^{\prime \prime}\right)$ and displacement amplitude of $\sim 70 \mathrm{~km}\left(0.10^{\prime \prime}\right)$ at that time $\left(t_{0}=11: 40: 29 \mathrm{UT}\right)$.

Figure 2 shows three temporal sequences of displacements, $D_{0}\left(t ; y_{1}\right), D_{0}\left(t ; y_{2}\right)$, and $D_{0}\left(t ; y_{3}\right)$, for Point-I, Point-II and Point-III on the spicule, where $y_{1}, y_{2}$ and $y_{3}$ are the longitudinal coordinates in the long slit and equal to $3^{\prime \prime}, 3.5^{\prime \prime}, 4.5^{\prime \prime}$, respectively. In Fig. 2, we find that points on the spicule oscillate along with the time, in addition to the average linear transverse motion across the central longitudinal axis of the long slit. Such average linear transverse motion of the point may be caused by the apparent turning of the spicule toward the photosphere or by long period waves acting on the spicule. The average linear transverse motion velocities for these three points from bottom to top are $3.1,4.3$ and $5.8 \mathrm{~km} \mathrm{~s}^{-1}$, respectively.

The linear transverse motion of the displaced point at the height $y, D_{y}(t)$, is a linear-fitting result of $D_{0}(t ; y)$ at the height $y$. We subtract $D_{y}(t)$ from $D_{0}(t ; y)$, and consider only the pure temporal oscillation at the height $y$, which we define as $D_{2}(t ; y)$. The upper panel of Fig. 3 shows the oscillation sequence for Point-II at the height of $y_{2}, D_{2}\left(t ; y_{2}\right)$, which can be fitted with a sinusoidal curve. FFT analysis is used to calculate the power spectrum
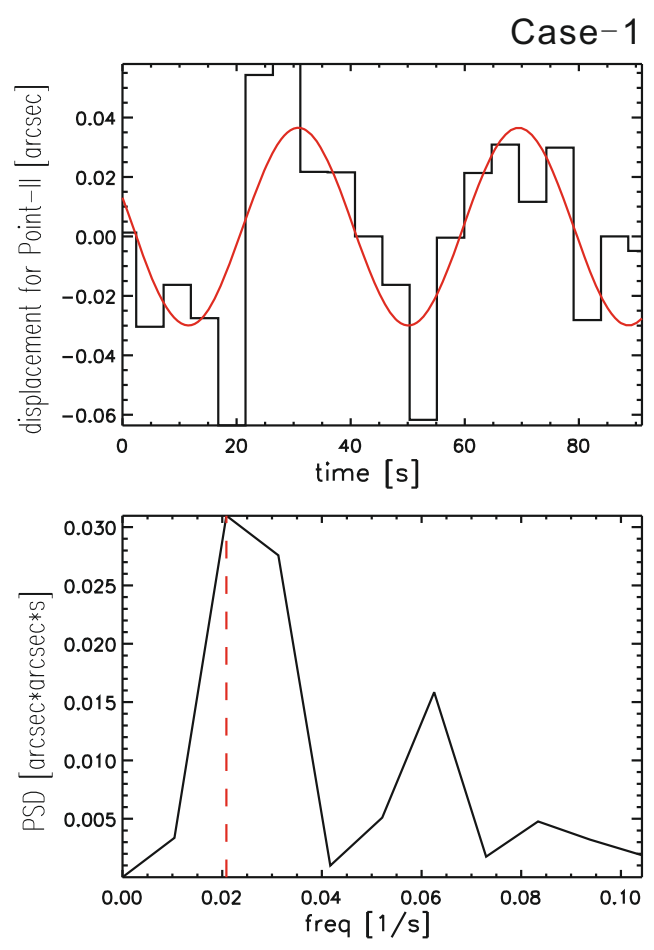

Fig. 3. Upper panel: Black line means the $D_{2}\left(t ; y_{2}\right)$ for Point-II. Red line is the fitting result with a sine function. Lower panel: Power spectrum density (PSD) of $D_{2}\left(t ; y_{2}\right)$ obtained with the FFT method.

density (PSD) shown in the lower panel of Fig. 3. It is found that the PSD has a maximum at the frequency of $0.021 \mathrm{~Hz}$, which corresponds to a period of $48 \mathrm{~s}$. This period is shorter than the lower limit of the period range for Alfvén waves carried by the spicules as reported by De Pontieu et al. (2007a). The displacement amplitude for the oscillation is estimated to be $\sim 36 \mathrm{~km}\left(0.05^{\prime \prime}\right)$ near the image pixel scale, and the velocity amplitude is estimated to be $\sim 4.7 \mathrm{~km} \mathrm{~s}^{-1}$. This velocity amplitude is smaller than the range from 10 to $25 \mathrm{~km} \mathrm{~s}^{-1}$, given by De Pontieu et al. (2007a).

We carry out the cross-correlation analysis of two oscillation sequences for two of the three points, $D_{2}\left(t, y_{1}\right)$ and $D_{2}\left(t, y_{2}\right)$, in order to judge the propagation direction of the oscillation as well as to estimate the phase speed. The upper left and upper right panels of Fig. 4 display the oscillation sequences for Point-I and Point-II, respectively. These two oscillations have a 

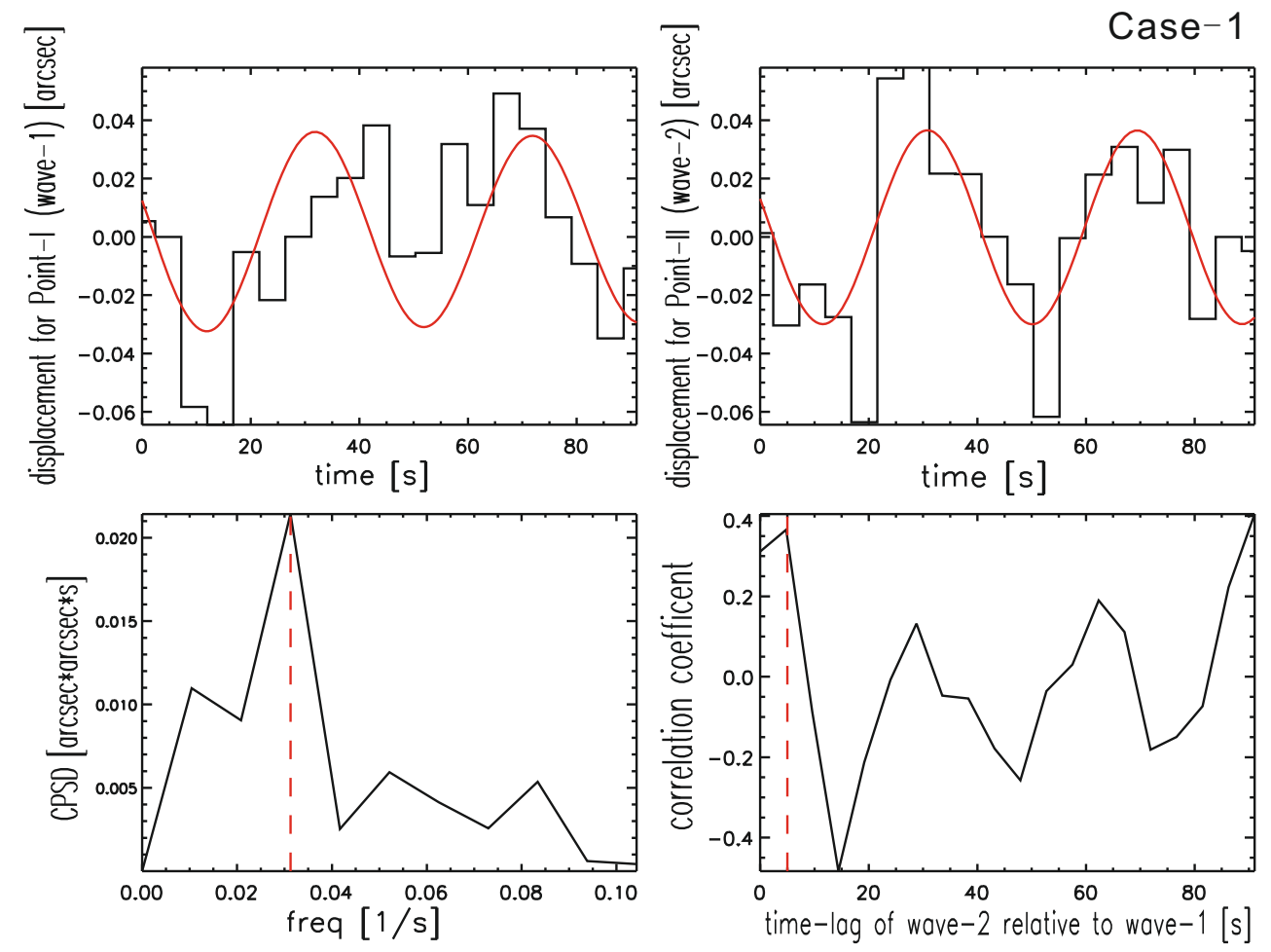

Fig. 4. Upper left panel: $D_{2}\left(t ; y_{1}\right)$ for Point-I (black line) and its sin-fit result (red line). Upper right panel: $D_{2}\left(t ; y_{2}\right)$ for Point-II (black line) and its sin-fit result (red line). Lower left panel: cross power spectrum density (CPSD) for the oscillations of Point-I and Point-II. Lower right panel: Correlation coefficient as a function of time lag between the oscillations of Point-I and Point-II.

maximum cross power spectrum density (CPSD) at a frequency of $0.031 \mathrm{~Hz}$, see the lower left panel of Fig. 4. The correlation coefficient between oscillations of Point-I and Point-II as a function of the time lag can be obtained by applying inverse-FFT method to the CPSD profile, and is plotted in the lower right panel of Fig. 4. The correlation coefficient has a maximum value at the time lag of $\sim 5 \mathrm{~s}$ showing that the oscillation for Point-II has a time lag of $\sim 5 \mathrm{~s}$ relative to the oscillation for Point-I, which indicates that the Alfvén wave propagates upwards with a phase speed of $\sim 75 \mathrm{~km} \mathrm{~s}^{-1}$ along the spicule. Please note that the phase speed may be larger than $75 \mathrm{~km} \mathrm{~s}^{-1}$ since we can not distinguish a time lag shorter than $5 \mathrm{~s}$, the interval between two exposures.

The upward propagation result is further confirmed in Fig. 5, by carrying out another cross-correlation analysis of $D_{2}\left(t ; y_{2}\right)$ and $D_{2}\left(t ; y_{3}\right)$, the oscillation sequences for Point-II and Point-III. It is found in the lower left panel of Fig. 5 that these two oscillations have a maximum CPSD at a frequency of $0.021 \mathrm{~Hz}$. As is shown in the lower right panel of Fig. 5, the correlation coefficient profile has a maximum at the time lag of $5 \mathrm{~s}$, the phase speed in the section between Point-II and Point-III is thus estimated to be $150 \mathrm{~km} \mathrm{~s}^{-1}$.

Three other cases with Alfvén waves propagating upward along the spicules are also analyzed in this paper. Here we give a brief introduction to these three cases in the main text. For more detailed illustrations, please see the supplemental materials in Appendix A. In the second case, the oscillation period of the Alfvén wave is estimated to be 37 s corresponding to a frequency of $0.027 \mathrm{~Hz}$. The amplitude of oscillating displacement is about $0.05^{\prime \prime}$, and the velocity amplitude is about $6.1 \mathrm{~km} \mathrm{~s}^{-1}$. The Alfvén wave carried by this spicule is also found to propagate upward with a phase speed range between 59 and $117 \mathrm{~km} \mathrm{~s}^{-1}$. The second spicule appears in wave-like shape with an apparent wavelength of $5800 \mathrm{~km}$ at 11:41:31 UT. In the third case, the displacement oscillation sequences appear to have a period of $45 \mathrm{~s}$ with three displacement humps in a time range of $100 \mathrm{~s}$. The displacement amplitudes are so large to reach $0.2^{\prime \prime}$, a value higher than the displacement amplitudes in the former two cases of Alfvén waves, which may be related with the apparent surge-up of materials at the foot of this spicule and its large inclination. The Alfvén wave propagates upward along this spicule with a phase speed of about $73 \mathrm{~km} \mathrm{~s}^{-1}$. There is no clear wavelike shape appearing on the third spicule. In the fourth case, the displacements oscillate with a period of $50 \mathrm{~s}$, and are greatly diminished from more than $0.1^{\prime \prime}$ to less than $0.1^{\prime \prime}$ after one period, which may indicate the damping of the Alfvén wave or the passing of an Alfvén wave packet. The Alfvén wave propagates upward along the fourth spicule with a phase speed range from 109 to $145 \mathrm{~km} \mathrm{~s}^{-1}$. The fourth spicule has a wave-like shape with an apparent wavelength of $5075 \mathrm{~km}$ at 11:49:11 UT. The second and the fourth spicules are visible at larger heights than the first and third spicules, and the lower sections of them are indiscernible. In Table 1, we list the characteristics of these four spicules with high-frequency Alfvén waves propagating upward on them.

\section{Summary and discussion}

We have found and analyzed four cases of dynamic spicules showing high-frequency transverse displacement oscillations with time-lagged phases at greater heights, which suggests a modulation of spicules by upward propagating high-frequency Alfvén waves. These Alfvén waves fluctuate at high frequencies $(>0.01 \mathrm{~Hz})$, and propagate upward with phase speeds in the range between 50 and $150 \mathrm{~km} \mathrm{~s}^{-1}$. The maximum apparent transverse velocities are estimated to be 4.7, 6.1, 18.1, and $20.8 \mathrm{~km} \mathrm{~s}^{-1}$, derived by using an expression of $D_{2, \max } \cdot 2 \pi / T$, where $D_{2, \max }$ is the maximum transverse displacement and $T$ is the wave period, which are compatible, respectively, with the values estimated directly from the time variations of the 

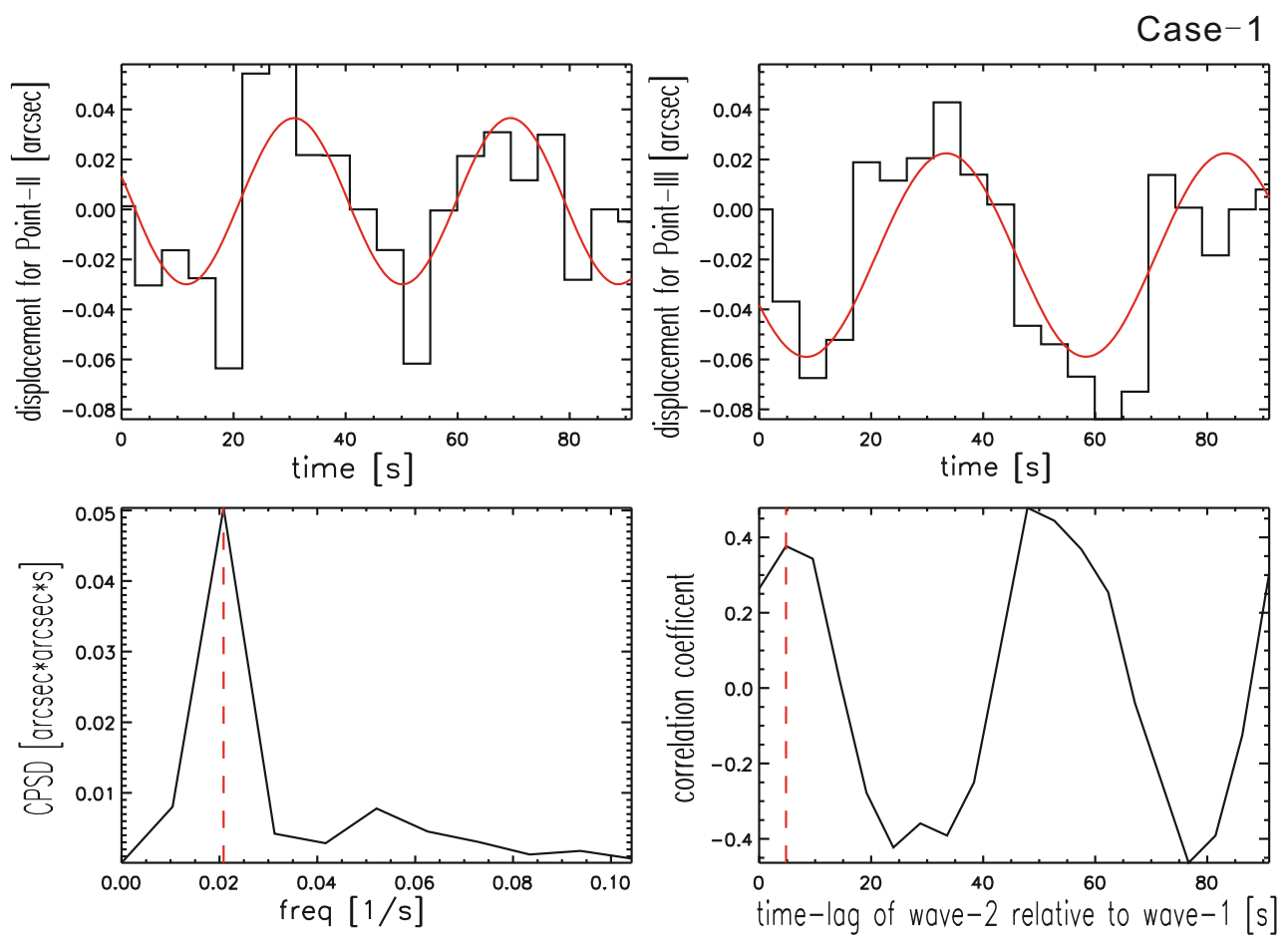

Fig. 5. Upper left panel: $D_{2}\left(t ; y_{2}\right)$ for Point-II (black line) and its sin-fit result (red line). Upper right panel: $D_{2}\left(t ; y_{3}\right)$ for Point-III (black line) and its sin-fit result (red line). Lower left panel: cross power spectrum density (CPSD) for the oscillations of Point-II and Point-III. Lower right panel: correlation coefficient as a function of time lag between the oscillations of Point-II and Point-III.

Table 1. Characteristics of four cases of spicule dynamics with Alfvén waves propagating upward along them ${ }^{a}$.

\begin{tabular}{cccccc}
\hline \hline Spicule number & Wave period & Phase speed & Velocity amplitude & Height & Inclination \\
\hline Spicule-1 & $48 \mathrm{~s}$ & $75-150 \mathrm{~km} \mathrm{~s}^{-1}$ & $4.7 \mathrm{~km} \mathrm{~s}^{-1}$ & $4.4 \mathrm{Mm}$ & $10^{\circ}$ \\
Spicule-2 & $37 \mathrm{~s}$ & $59-117 \mathrm{~km} \mathrm{~s}^{-1}$ & $6.1 \mathrm{~km} \mathrm{~s}^{-1}$ & $5.8 \mathrm{Mm}$ & $29^{\circ}$ \\
Spicule-3 & $45 \mathrm{~s}$ & $73 \mathrm{~km} \mathrm{~s}^{-1}$ & $18.1 \mathrm{~km} \mathrm{~s}^{-1}$ & $1.5 \mathrm{Mm}$ & $43^{\circ}$ \\
Spicule-4 & $50 \mathrm{~s}$ & $109-145 \mathrm{~km} \mathrm{~s}^{-1}$ & $20.8 \mathrm{~km} \mathrm{~s}^{-1}$ & $7.3 \mathrm{Mm}$ & $30^{\circ}$ \\
\hline
\end{tabular}

${ }^{a}$ Note: all these characteristics are obtained from spicule dynamics in the projected plane. The "height" in the fifth column is the Point-III height above the limb. The "inclination" in the sixth column is the spicule inclination angle off the direction normal to the solar limb.

transverse displacements. The relatively smaller apparent velocity amplitudes for the Alfvén waves on the first and second spicules may be caused by the projection effect. They are still discernable after the elimination of jitter and slow drift on the image time series as well as the large-amplitude low-frequency transverse motion of the spicule.

The dynamics of the third spicule shows an obvious surge-up at the foot, and the spicule is highly inclined, which may explain the observed large transverse velocity amplitude of Alfvén wave on it. The fourth spicule may be modulated by an Alfvén wave packet, and thus experiences a sequence of transverse oscillation with amplitude being large at the beginning and decreasing soon after one period. Damping of the Alfvén waves may be another possibility for the decrease of their velocity amplitudes. The heights above the limb in the projected plane for the four spicules, where we studied the transverse displacement oscillation, are maximally located at 4.4, 5.8, 1.5, and 7.3 Mm, respectively. We did not check observationally if these high-frequency waves exist in more extended spicule structures, say above $10 \mathrm{Mm}$. However, from theoretical consideration, it appears that for high-frequency waves it may be easier to propagate outward without reflection. We do not see noticeable growth or drop in wave displacement amplitude on the first three spicules except for the dynamics of the fourth spicule, which shows a growth in amplitude with increasing height and a damping in amplitude with increasing time at the same height.

To our knowledge, this is the first observation of an upward propagating Alfvén wave with a frequency higher than $0.01 \mathrm{~Hz}$ in the chromosphere and transition region. The Alfvén waves with a period of $50 \mathrm{~s}$ may be damped by cyclotron resonance at about $60 R_{\mathrm{s}}$, which is the mechanism used in models for the solar wind heating (Tu \& Marsch 1997; Marsch \& Tu 1997). Such high-frequency Alfvén wave may undergo some damping in the corona if the nonlinear transverse cascade (Cranmer \& van Ballegooijen 2005) or the mode conversion to slow magneto-acoustic shock (Suzuki \& Inutsuka 2005) actually take place. However, the turbulence cascading in the solar corona may be weak (Tu \& Marsch 1997), therefore it is possible these waves will not be dissipated quickly within $60 R_{\mathrm{s}}$. The question as to how such waves are dissipated is still open. The velocity amplitude reported here is less than the mean velocity amplitude used in the model by Tu \& Marsch (1997). This smallness is natural, since the former has a narrow frequency band, which is embedded in the wide frequency band of the latter, and moreover the observation involves a projection effect. Due to the lack of three dimensional imaging of the dynamic wavelike spicules, we only consider, in a plane perpendicular to the LOS, the distance between points as well as the displacements of 

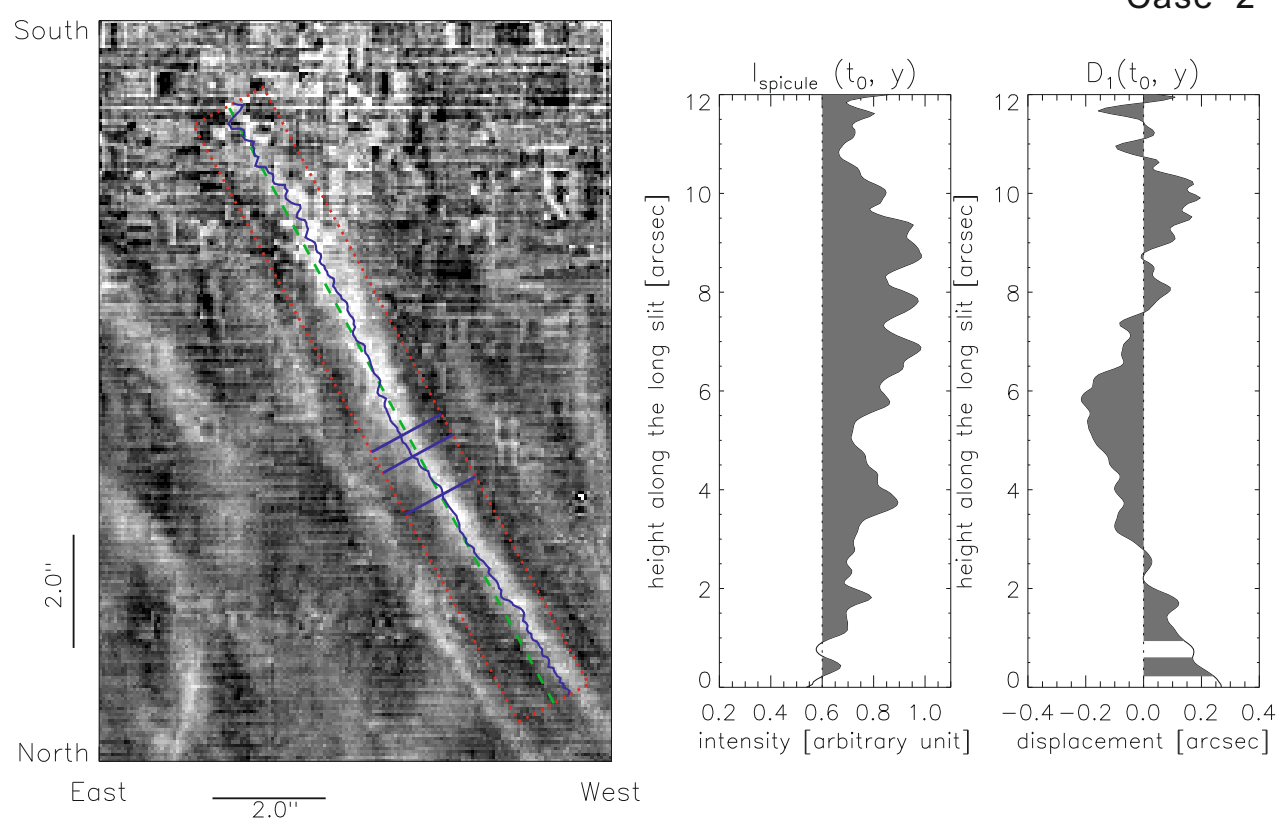

Fig. A.1. The spatial profiles of intensity and displacement of the spicule at $t_{0}=11: 41: 31$ UT for Case- 2 .

the points. This consideration may underestimate to some extent the phase speed and velocity amplitude of the Alfvén wave, accordingly. To accurately calculate the properties of Alfvén wave, imaging of the spicule from many more visual angles is required.

At present, there are three possible mechanisms to generate the Alfvén waves, of which horizontal random walk on the photosphere of the foot-point (van Ballegooijen et al. 1998) of a field line and photospheric 5-min oscillation as well as chromospheric 3-min oscillation (supporting Online Materials in De Pontieu et al. 2007a) are both considered to be responsible for the low-frequency Alfvén waves, while the third mechanism namely small-scale magnetic reconnection is expected to excite the high-frequency Afvén waves as suggested by Axford et al. (1999). However, up to now, there is still a lack of direct observational connection between the three possible mechanisms and the observed waves. The Alfvén waves with the short wavelength and period discussed in this paper may originate from magnetic reconnection, whereas the low-frequency Alfvén waves reported by De Pontieu et al. (2007a) were argued to be caused by the 5-min p-mode and 3-min chromospheric oscillation. These mechanisms may be related with the spicule dynamics which is found to consist of two types: type-I spicules that are probably driven by shock wave and have lifetimes of 3-7 min, and type-II spicules that are probably driven by magnetic reconnection and have lifetimes of 10-150 s (De Pontieu et al. 2007b).

The wave fluctuation on the spicule may be called the fast "kink" mode wave, if the spicule can be described as a stable flux tube in the non-uniform medium. The "kink" mode wave will finally evolve into Alfvén wave after its propagation upward into the more homogeneous medium (Cranmer et al. 2007). The apparent thicknesses of the spicules we studied are wider than $120 \mathrm{~km}$, corresponding to the diffraction limit of SOT in $3968 \AA$. It is possible that such spicules may contain some very fine threads thinner than $120 \mathrm{~km}$. These possibly existent threads seem to be affected by the same wave fluctuation and show consistent dynamics. It seems difficult for these thin threads to mimic the observed high-frequency dynamic behavior if they were moving out of phase in low-frequency large-amplitude oscillations, because they would then spin together and separate from time to time, and so finally destruct the spicule.

In this paper, we only presented four examples of upward propagating Alfvén waves along the spicules. These four examples show that high-frequency Alfvén waves do exist in the lower solar atmosphere. However it is not easy to pick out more spicules satisfying the analysis requirements from our dataset, since spicules rarely exist individually without overlapping or interacting with other spicules, and since very few spicules can be visible for a time only as long as two wave periods. Because of the limitation of the observational conditions, we cannot judge how much energy they can carry. To explore experimentally how much energy the high-frequency waves may contain, and to see if they can contribute to the corona heating and wind acceleration is a new task suggested by this study. A statistical study of the upward propagating Alfvén waves carried by more spicules is needed in the future work, in order to give a quantitative estimate of the energy flux carried by these waves.

Acknowledgements. Hinode is a Japanese mission developed and launched by ISAS/JAXA, with NAOJ as domestic partner and NASA and STFC (UK) as international partners. It is operated by these agencies in co-operation with ESA and NSC (Norway). SOT onboard Hinode was developed jointly by NAOJ, LMSAL, ISAS/JAXA, NASA, HAO and MELCO. We thank the SOT team for making their data public available on the internet. This work at Peking University is supported by the National Natural Science Foundation of China under Contract Nos. 40874090 and 40336053 and the Beijing Education Project XK100010404. He Jiansen's work is also supported by "China Postdoctoral Science Foundation funded project".

\section{Appendix A: Another three cases for upward propagation of Alfvén waves}

In this Appendix, we show another three cases of upwardpropagating Alfvén waves. The figure formats in these three cases are similar to those in the first case. Several key characteristics (wave period, phase speed, and velocity amplitude) for the Alfvén waves on these three spicules are listed in Table 1. Please note that the number of clear cases found in our analyzed dataset is rather limited. 
Case-2
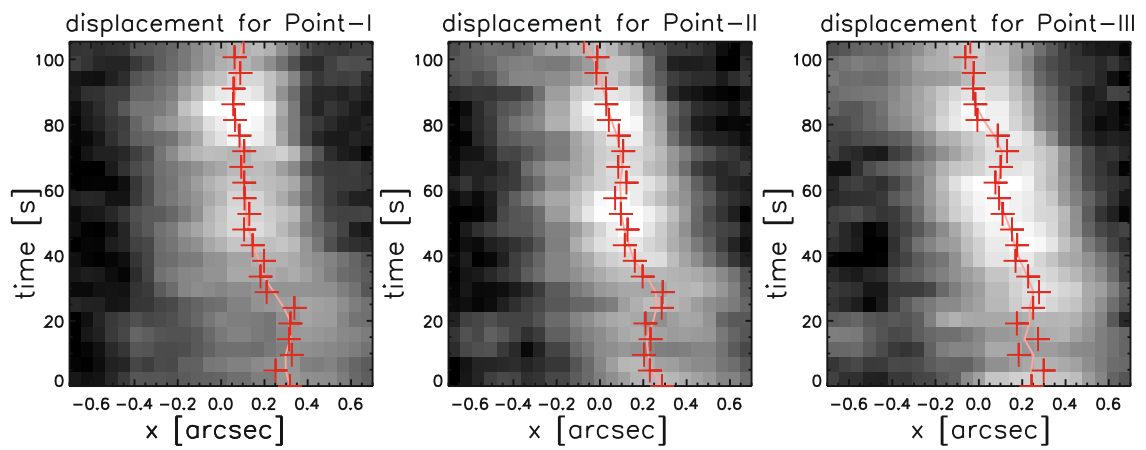

Fig. A.2. Profiles of $D_{0}(t ; y)$ for Point-I, Point-II, and Point-III on the spicule for Case-2.

Case-2
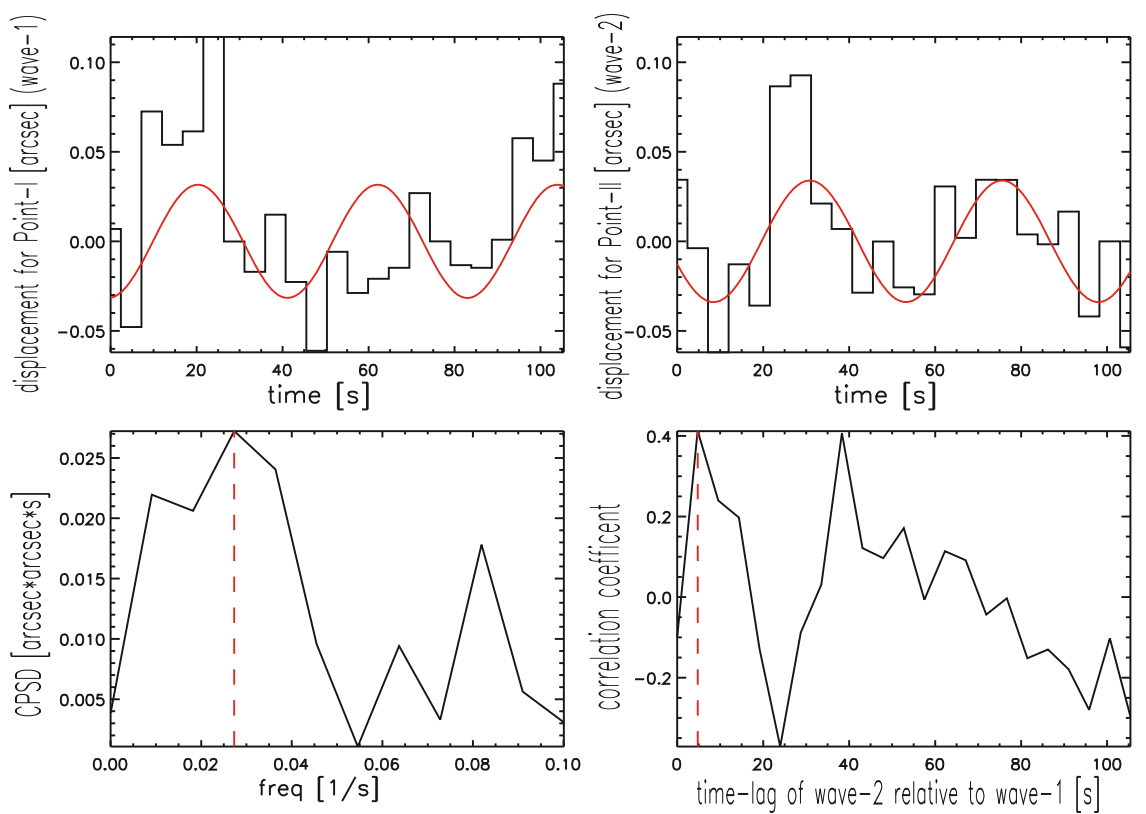

Fig. A.3. Correlation analysis result between $D_{2}\left(t ; y_{1}\right)$ for Point-I and $D_{2}\left(t ; y_{2}\right)$ for Point-II for Case-2.

Case-2
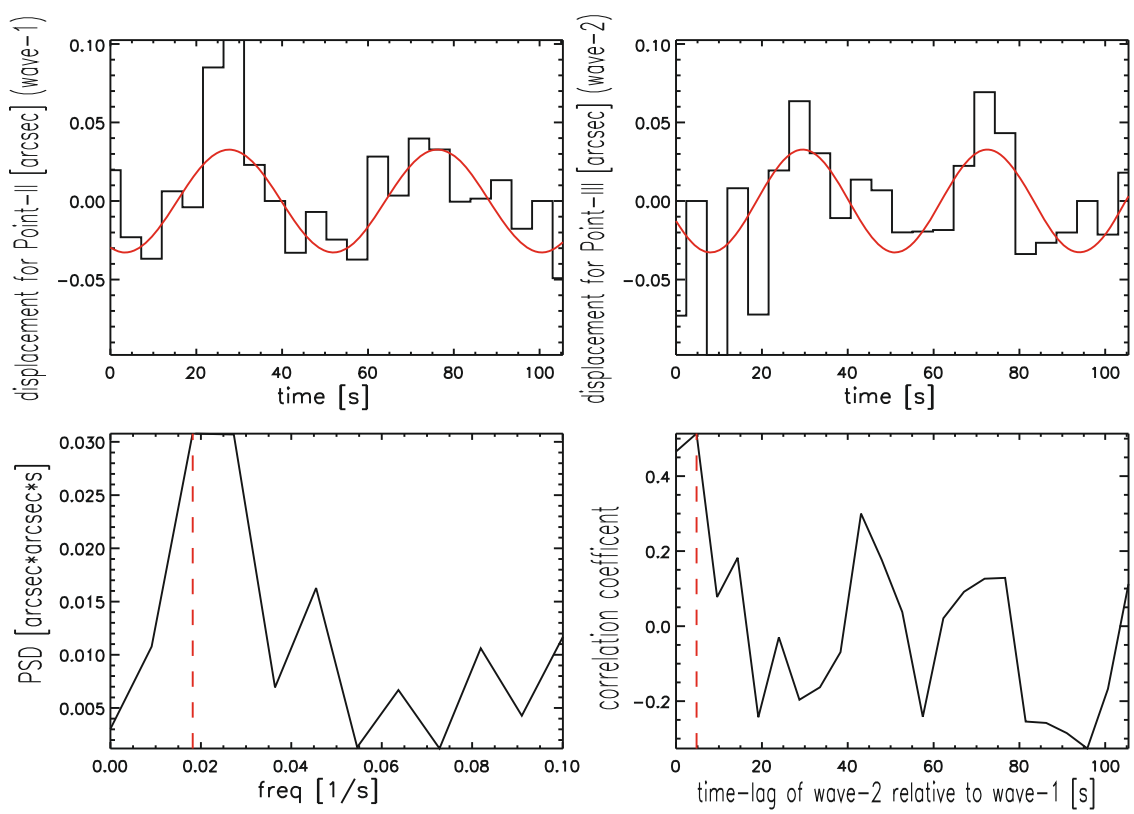

Fig. A.4. Correlation analysis result between $D_{2}\left(t ; y_{2}\right)$ for Point-II and $D_{2}\left(t ; y_{3}\right)$ for Point-III for Case-2. 

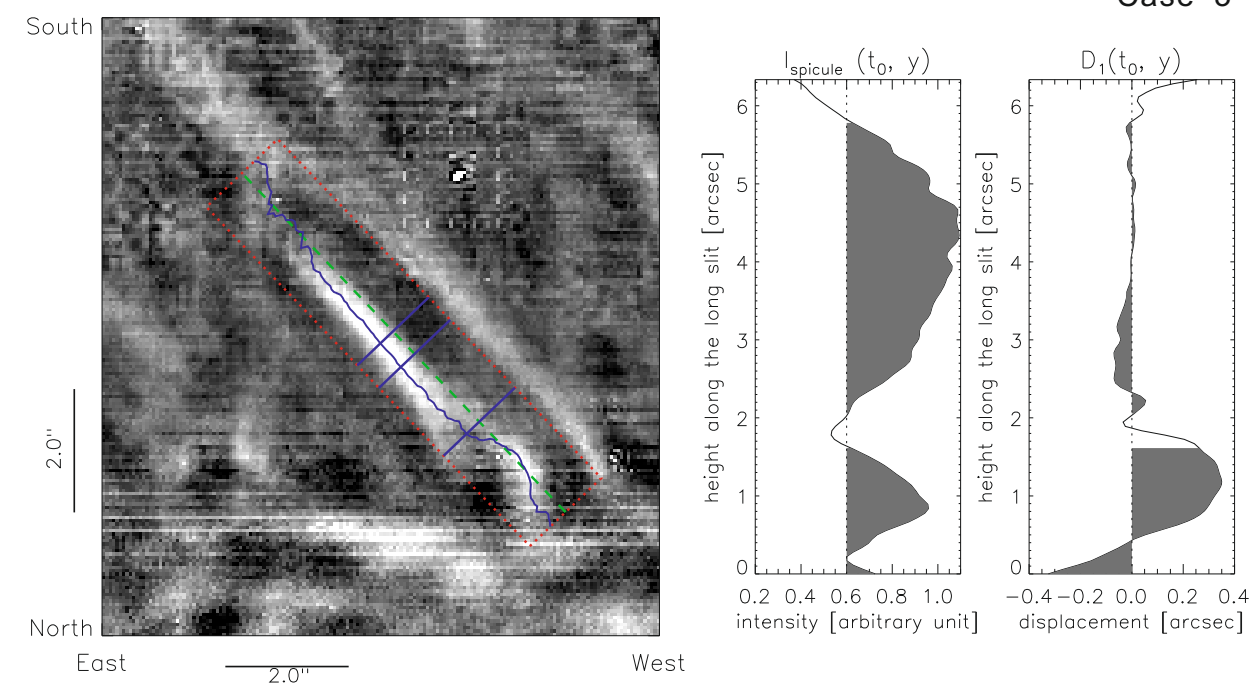

Fig. A.5. The spatial profiles of intensity and displacement of the spicule at $t_{0}=11: 33: 56$ UT for Case-3.

Case-3
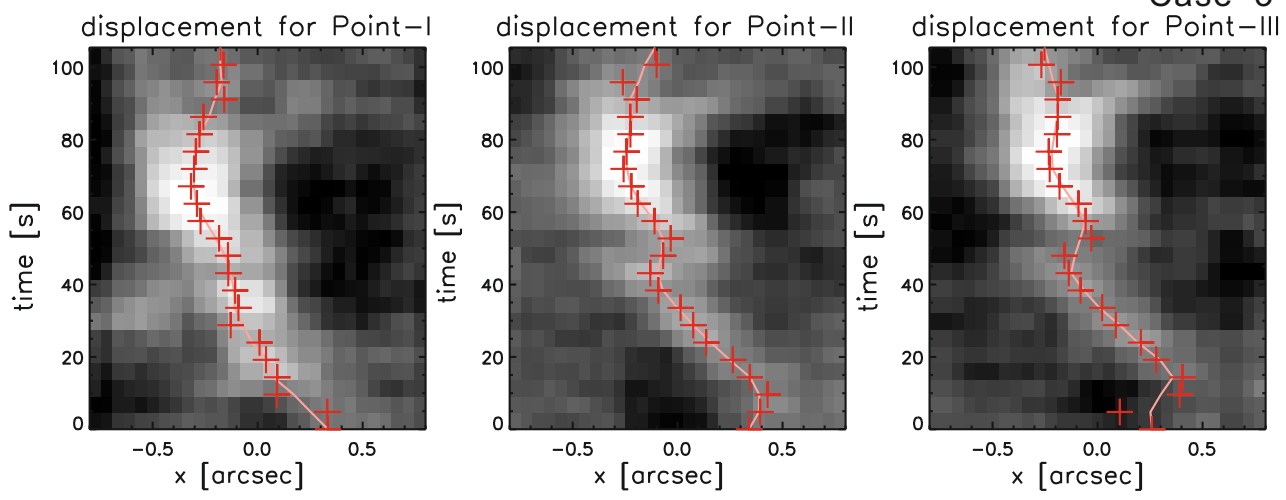

Fig. A.6. Profiles of $D_{0}(t ; y)$ for Point-I, Point-II, and Point-III on the spicule for Case-3.
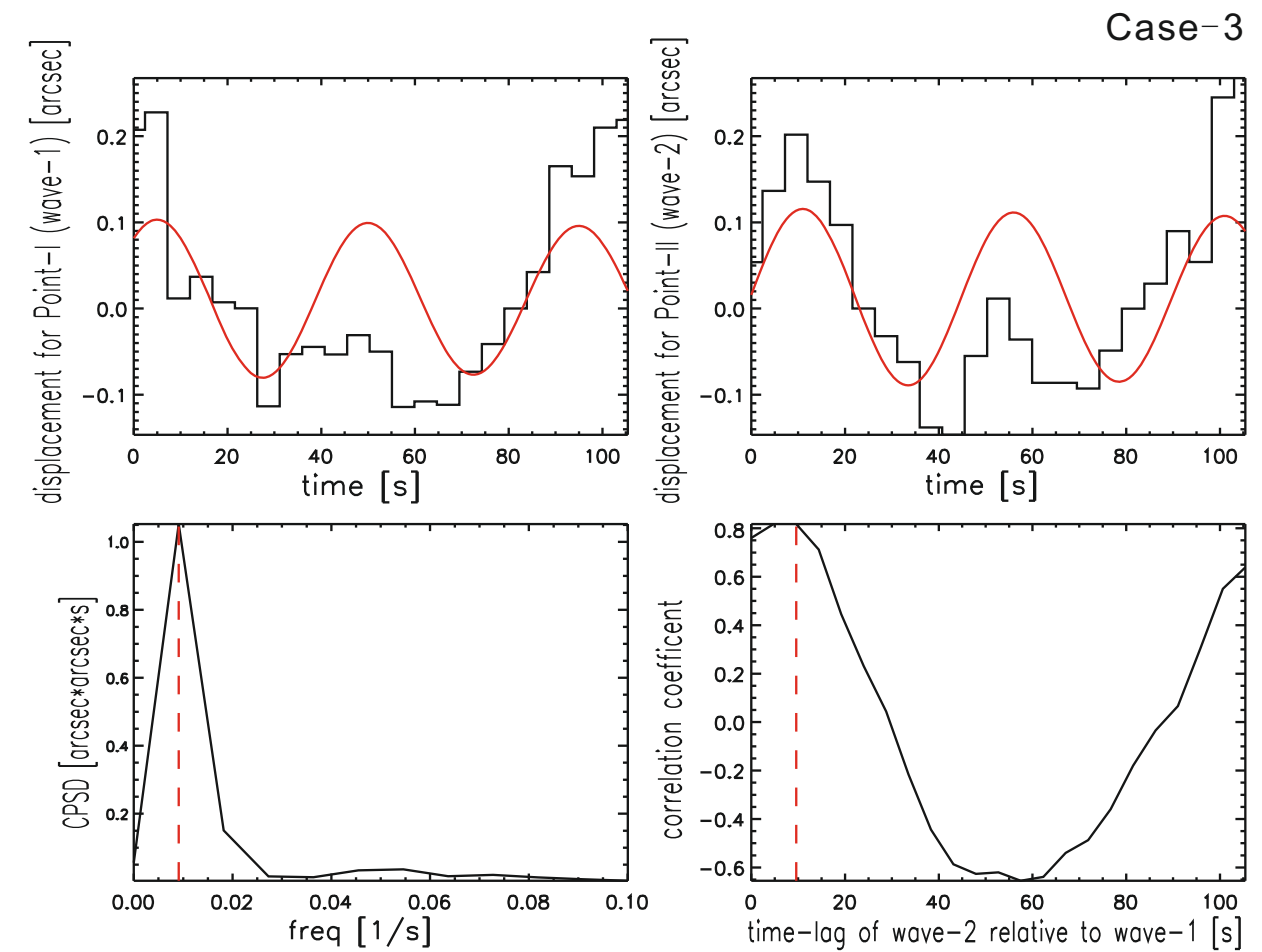

Fig. A.7. Correlation analysis result between $D_{2}\left(t ; y_{1}\right)$ for Point-I and $D_{2}\left(t ; y_{2}\right)$ for Point-II for Case-3. 

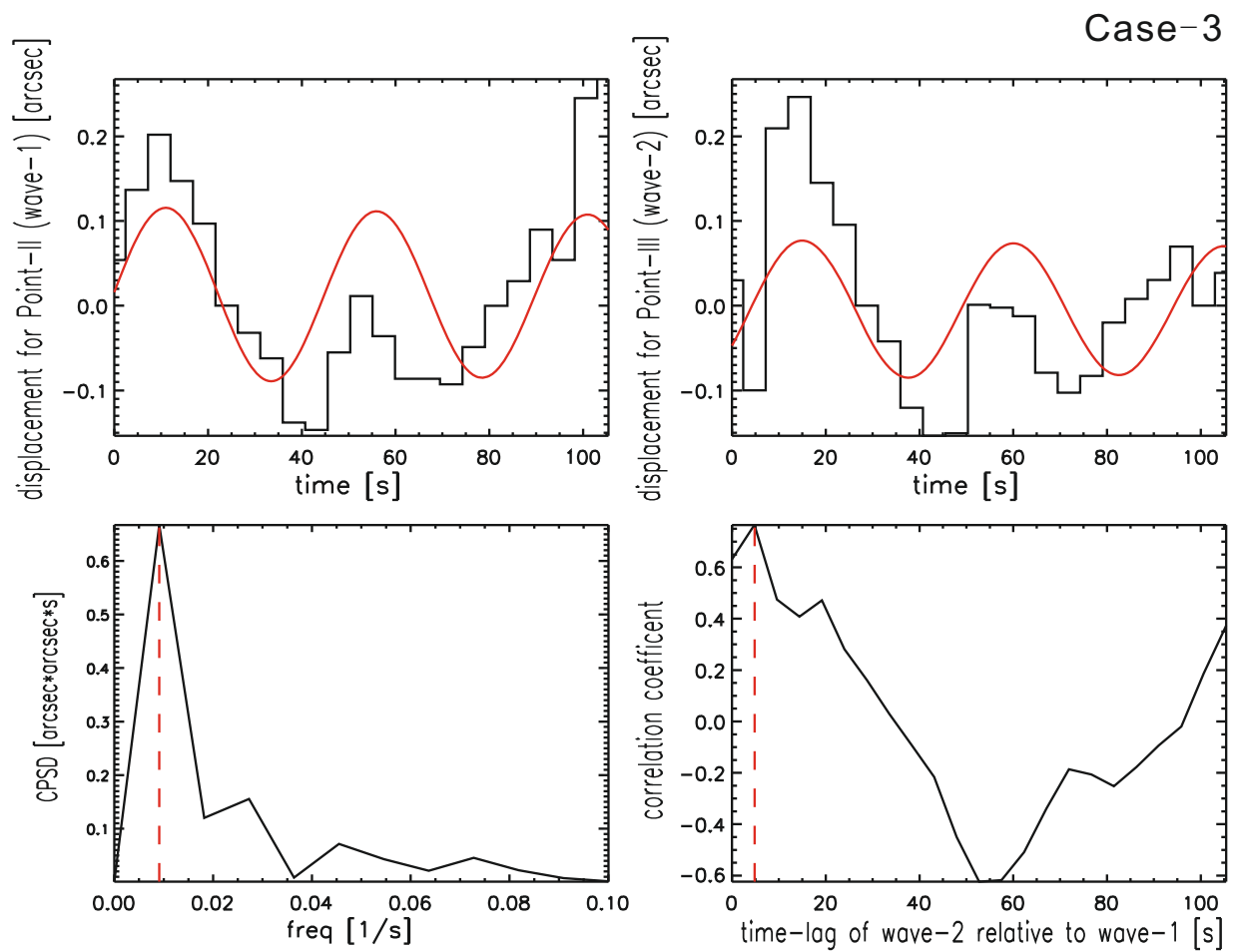

Fig. A.8. Correlation analysis result between $D_{2}\left(t ; y_{2}\right)$ for Point-II and $D_{2}\left(t ; y_{3}\right)$ for Point-III for Case-3.
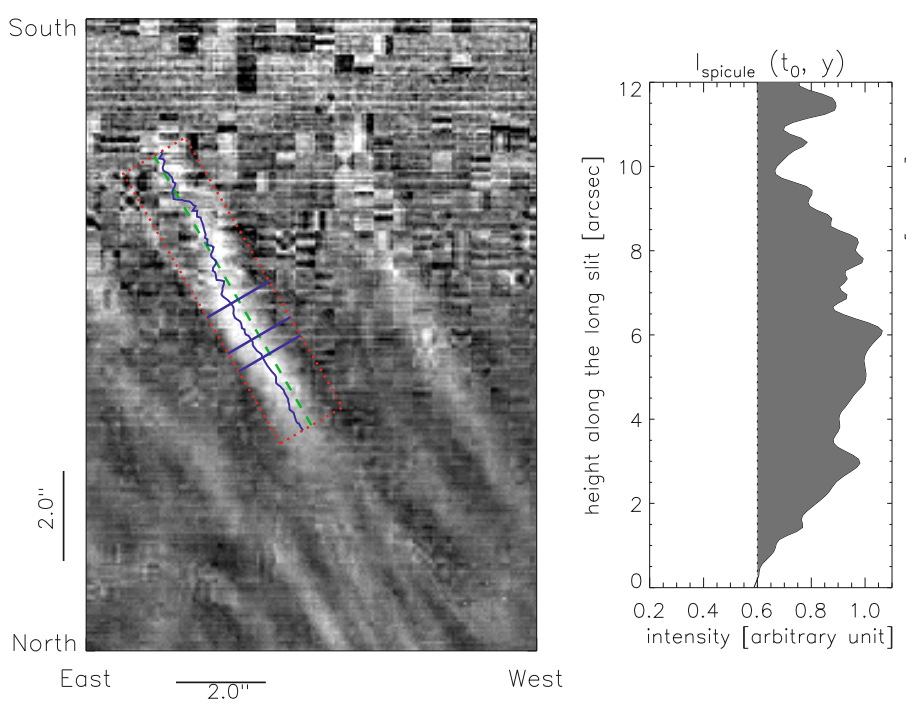

Case -4

Fig. A.9. The spatial profiles of intensity and displacement of the spicule at $t_{0}=11: 49: 11$ UT for Case- 4 .

Case -4
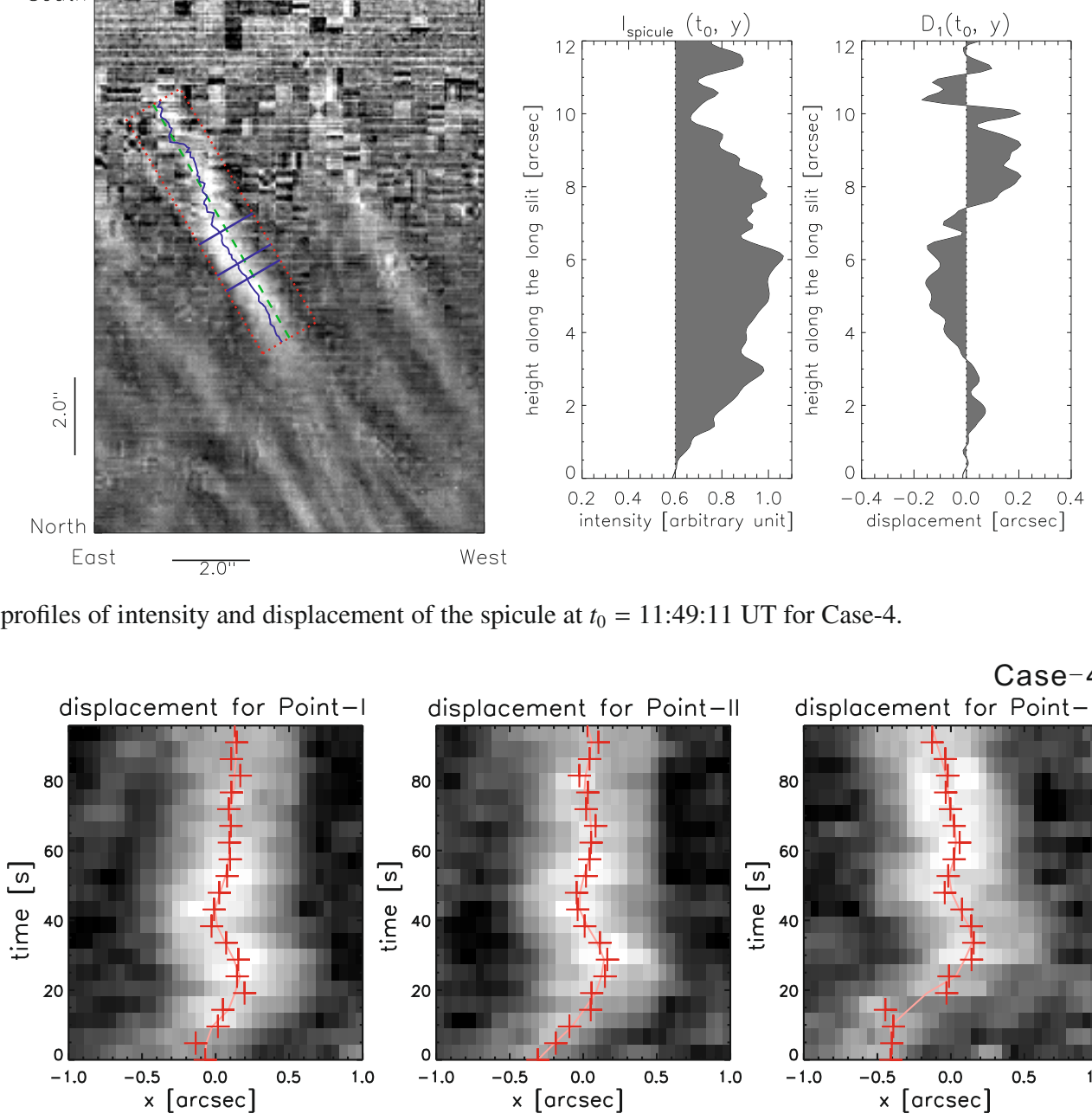

or Point-III

Fig. A.10. Profiles of $D_{0}(t ; y)$ for Point-I, Point-II, and Point-III on the spicule for Case- 4 .

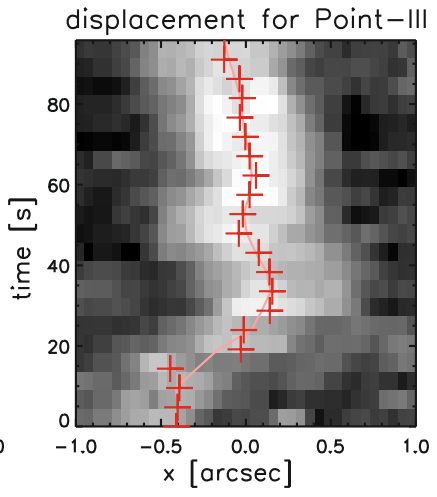



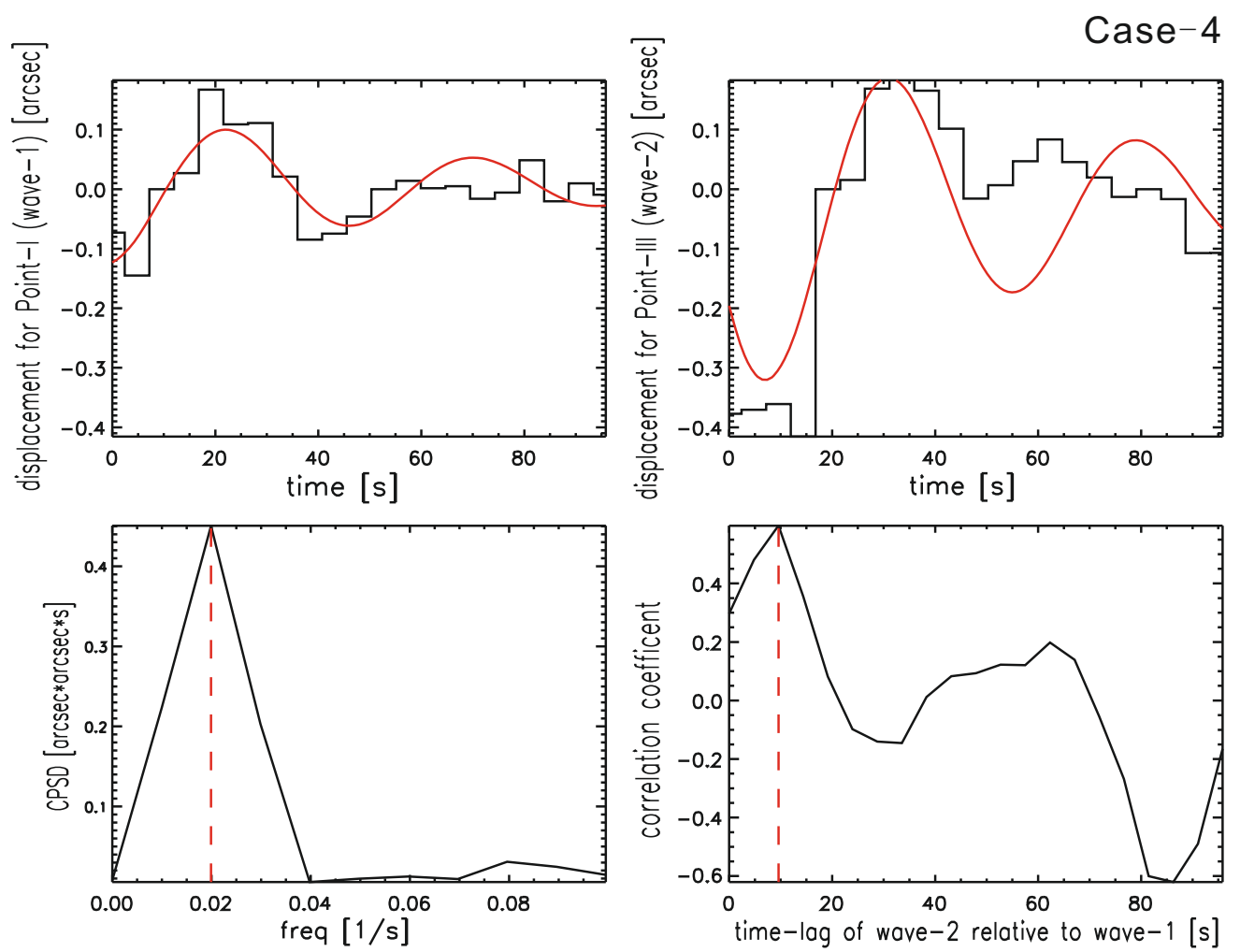

Fig. A.11. Correlation analysis result between $D_{2}\left(t ; y_{1}\right)$ for Point-I and $D_{2}\left(t ; y_{3}\right)$ for Point-III for Case-4.
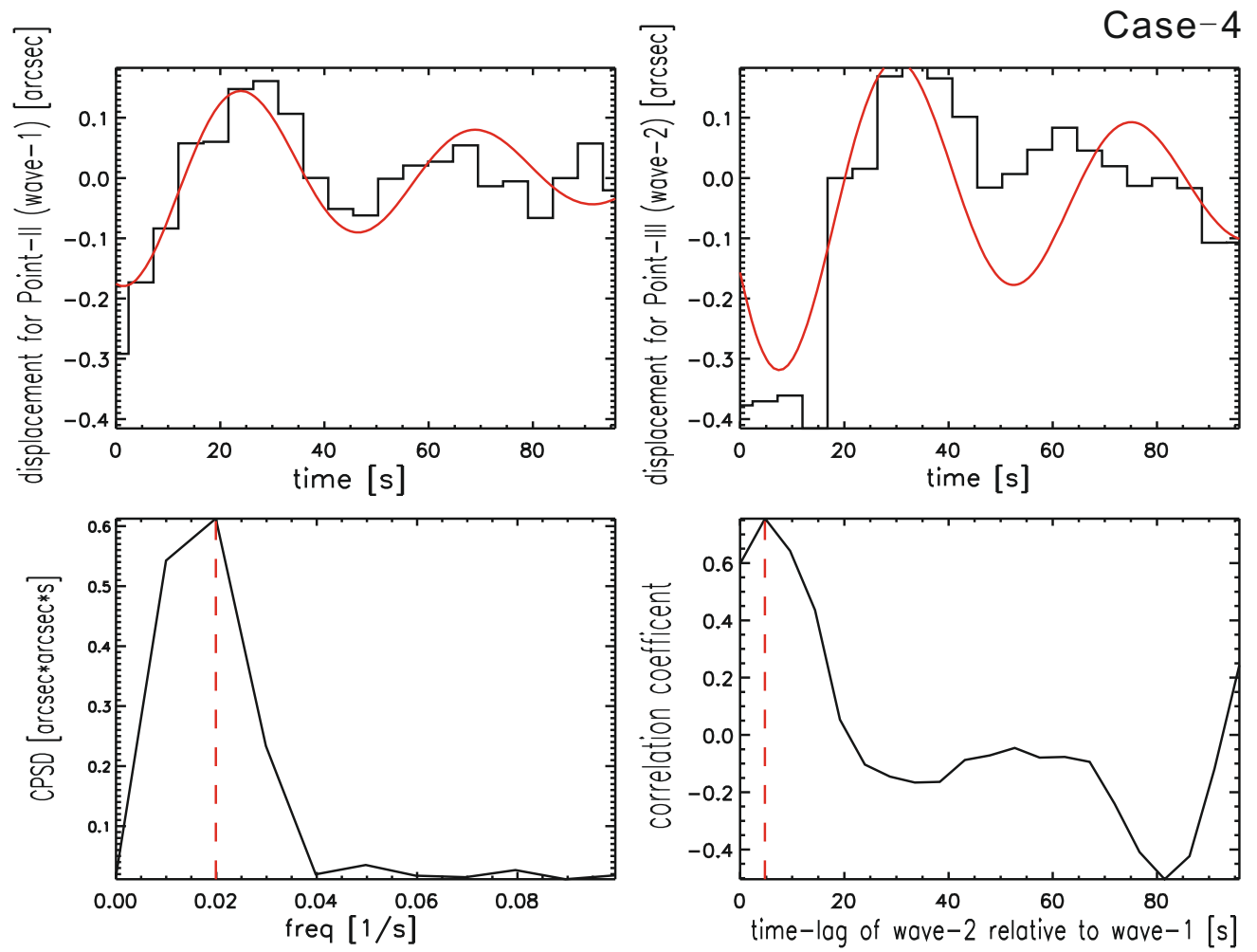

Fig. A.12. Correlation analysis result between $D_{2}\left(t ; y_{2}\right)$ for Point-II and $D_{2}\left(t ; y_{3}\right)$ for Point-III for Case-4. 


\section{References}

Axford, W. I., McKenzie, J. F., Sukhorukova, G. V., et al. 1999, Space Sci. Rev., 87,25

Cranmer, S. R., \& van Ballegooijen, A. A. 2005, ApJS, 156, 265

Cranmer, S. R., van Ballegooijen, A. A., \& Edgar, R. J. 2007, ApJS, 171, 520

De Pontieu, B., McIntosh, S. W., Carlsson, M., et al. 2007a, Science, 318, 1574

De Pontieu, B., McIntosh, S., Hansteen, V. H., et al. 2007b, PASJ, 59, 655

He, J.-S., Tu, C.-Y., \& Marsch, E. 2008, Sol. Phys., 250, 147

Hollweg, J. V. 2000, J. Geophys. Res., 105, 15699

Hollweg, J. V., \& Isenberg, P. A. 2002, J. Geophys. Res., 107, 1147

Kosugi, T., Matsuzaki, K., Sakao, T., et al. 2007, Sol. Phys., 243, 3

Koutchmy, O., \& Koutchmy, S. 1989, in High spatial resolution solar observations, ed. O. von der Luehe, 217
Marsch, E., \& Tu, C.-Y. 1997, Sol. Phys., 176, 87

Matthaeus, W. H., Zank, G. P., Oughton, S., Mullan, D. J., \& Dmitruk, P. 1999, ApJ, 523, L93

Morgan, H., Habbal, S. R., \& Woo, R. 2006, Sol. Phys., 236, 263

Shimizu, T., Nagata, S., Tsuneta, S., et al. 2008, Sol. Phys., 249, 221

Suzuki, T. K., \& Inutsuka, S.-I. 2005, ApJ, 632, L49

Tomczyk, S., McIntosh, S. W., Keil, S. L., et al. 2007, Science, 317, 1192

Tsuneta, S., Ichimoto, K., Katsukawa, Y., et al. 2008, Sol. Phys., 249, 167

Tu, C.-Y., \& Marsch, E. 1995, Space Sci. Rev., 73, 1

Tu, C.-Y., \& Marsch, E. 1997, Sol. Phys., 171, 363

Tu, C.-Y., \& Marsch, E. 2001, J. Geophys. Res., 106, 8233

Tu, C.-Y., Marsch, E., Wilhelm, K., \& Curdt, W. 1998, ApJ, 503, 475

van Ballegooijen, A. A., Nisenson, P., Noyes, R. W., et al. 1998, ApJ, 509, 435

Wilhelm, K., Marsch, E., Dwivedi, B. N., \& Feldman, U. 2007, Space Sci. Rev.,

133,103 